\title{
LOS MECANISMOS MULTILATERALES COMO INSTRUMERTO DE LA PROYECCIÓn ESPAก̃OLA EN EL EXTERIOR
}

\section{Multilateral mechanisms as tools for Spanish projection abroad}

\author{
Gracia Abad \\ Universidad Nebrija \\ Área de Relaciones Internacionales
}

gabad@nebrija.es

Históricamente España ha mantenido una presencia discreta en las organizaciones internacionales, que se convirtió en casi testimonial en las décadas que siguen a la guerra civil. Esta tendencia comenzó a cambiar en los años 70 con la llegada de la Transición Política. Desde entonces, España ha tratado de incrementar su presencia en organizaciones internacionales y mecanismos multilaterales como forma de ejecutar su política exterior. Este proceso forma parte de un esfuerzo para generar "poder inteligente" que ayude a la consecución de los objetivos de la política exterior española.

España, política exterior, organización internacional, multilateralismo, "Poder Inteligente". Spain, foreign policy, International organization, multilateralism, Smart Power.

Q Key mords

Recibido: 31/07/2014. Aceptado: 25/08/2014

Fechas

Historically, Spain has maintained a discrete presence in International Organizations, which became almost symbolic during the decades after the civil war. This trend began to change at the early 70 s with the arrival of the Spanish political transition. Since then, Spain has sought to increase its presence in International organizations and multilateral fora as a way to run its foreign policy. This process is part of an effort to generate "smart power" so that it can help to achieve the aims of the Spanish foreign policy. 


\section{Introducción}

Una mirada a la presencia que históricamente ha tenido España en los mecanismos multilaterales en general y en las Organizaciones Internacionales en particular lleva fácilmente a una conclusión: el papel español en esos mecanismos ha sido tradicionalmente reducido y, en consecuencia, la capacidad española para hacer valer sus preferencias recurriendo a esos mecanismos muy limitada.

Sin embargo, un análisis algo más sosegado de la cuestión nos permite detectar no sólo elementos de continuidad sino también ciertas discontinuidades en relación con el papel y presencia de España en los mecanismos multilaterales.

Así, cabe establecer dos fases diferenciadas en lo que hace a la presencia española en marcos multilaterales en general y Organizaciones Internacionales en particular:

1. Una primera fase que se extiende desde el surgimiento de las organizaciones internacionales a finales del siglo XIX y se prolonga hasta los años finales del Franquismo

2. Una segunda fase que se abriría tímidamente en esos años finales de la dictadura franquista y, definitivamente, con la transición a la democracia y que se prolonga hasta nuestros días

Si en el primero de los periodos mencionados la línea dominante es la de la escasa presencia española, casi marginación, en los foros multilaterales, en la segunda fase considerada, la dinámica cambia por completo y, progresivamente, a la incorporación y asentamiento de España en las Organizaciones Internacionales y procesos multilaterales de distinto tipo sigue su creciente protagonismo en el seno de esos procesos e, incluso su liderazgo en el establecimiento de algunos de ellos.

Así, en las páginas que siguen trataremos de explicar la evolución seguida por la presencia española en los distintos tipos de marcos multilaterales, analizando tanto ambas etapas como los elementos caracterizadores de la segunda etapa, cuestión prácticamente inexistente en la literatura que sí documenta, en cambio, notablemente, los perfiles del primero de los periodos mencionados.

En este sentido, trataremos de mostrar el lugar que ocupa la participación de España en mecanismos multilaterales en el conjunto de la política exterior española (Montobbio, 2006) en este segundo periodo. Al respecto, plantearemos cómo en el segundo de los periodos mencionados España que, en tanto que potencia media, cuenta con unos recursos de "poder duro" limitados, debe tratar de alcanzar sus objetivos en materia de política exterior por medio del recurso al "poder inteligente", esto es, combinando hábilmente sus recursos tanto de "poder duro" como de "poder blando" en una estrategia que resulte verdaderamente efectiva (Nye, 2011). En ese marco, la participación española en organizaciones y el fomento de distintos procesos multilaterales, dinámicas crecientemente presentes en el periodo del que hablamos, no son sino un elemento - pero un elemento destacado- del esfuerzo de generación de un "poder inteligente" español o, lo que es lo mismo, del diseño de una estrategia efectiva que combinando elementos de "poder duro" y "poder blando" permita alcanzar los objetivos de la política exterior española.
España debe tratar de alcanzar sus objetivos en materia de política exterior por medio del "poder inteligente" 


\section{La presencia española en mecanismos multilaterales hasta la transición a la democracia}

Durante el siglo XIX España se va integrado progresivamente a las organizaciones internacionales -de carácter técnico sobre todo en un primer momento- que van surgiendo. En esa misma línea, España también tomará parte en la primera organización de carácter global, la Sociedad de Naciones y en la OIT (Granell, 2011)

En el caso de la Sociedad de Naciones, España no participa en el Tratado de Versalles como consecuencia de su estatuto de neutralidad en la I Guerra Mundial, pero en abril de 1919 se propuso que fuera admitida, algo que ocurriría. De hecho, fue incluida incluso en el Comité Ejecutivo de la Organización. Con todo, la participación en la SdN fue limitada, como muestra el hecho de no haber contado nunca, por ejemplo con un representante permanente ante la Sociedad de Naciones. Algo que en realidad no era algo característico sólo de la participación española en la SdN sino que cabe decir que cuanto mayor fuera el calado político de las cuestiones a decidir o de la organización de que se trataba, menor era el papel que, por lo general, jugaba España. En realidad no era extraño si se tiene en cuenta que ya había estado ausente del Congreso de Viena en 1815 y que espera hasta 1817 para unirse al Concierto Europeo. (Granell, 2011).

Con esas pautas de actuación no resulta sorprendente que, como recuerda Francesc Gravell hasta 1900 solamente 6 de los congresos internacionales celebrados tuvieran como marco España y que si tomamos como fecha de referencia 1914, la cifra sigue sin pasar de 30 (Granell, 2011).

Con todo, a comienzos del siglo XX España fue sede de dos organizaciones internacionales -Liga Internacional de Aviadores e Instituto Económico Americano-Casa de América-, organizó la Exposición Universal de Barcelona en 1898 y también las ferias internacionales de Sevilla y Barcelona (Granell, 2011).

Sin embargo, la guerra civil española y las cuatro décadas del régimen del General Franco supondrían una ruptura incluso con esa mínima inserción internacional que no volverá a restaurarse, al menos completamente, hasta la transición a la democracia. Así, el 8 de mayo de 1939, en línea con lo que hacen las otras potencias del Eje -Alemania, Italia, Japón-, España se retira de la Sociedad de Naciones ${ }^{1}$ (Granell, 2011) y, cuando tras la II Guerra Mundial se crea una nueva organización de seguridad colectiva, las Naciones Unidas, España no sería ni siquiera invitada a formar parte. En efecto, ya en la reunión celebrada en Postdam en 1945 los participantes se habían mostrado contrarios a la admisión de España por contar con un gobierno que había contado con el apoyo de las potencias del Eje (Fernández Liesa, Manero \& Quispe, 2014).

De este modo, durante cuatro décadas España se limitaría a mantener una posición de perfil bajo, casi testimonial -más acusada al principio-, en algunos mecanismos multilaterales (en esos años, fundamentalmente organizaciones internacionales), estando completamente ausente de otros. Una posición que, sin embargo, no es tanto el resultado de la voluntad española como de la imposición externa fruto del peculiar régimen político de la España de la época. Así, el 12 de diciembre de 1946 la Resolución 39 (1) de la Asamblea General de las Naciones

\section{España forma parte de todas las grandes Organizaciones Intemacionales}


Unidas condenaba a España al aislamiento al pedir expresamente la exclusión del gobierno de Franco de las organizaciones internacionales y conferencias (Granell, 2011) así como la retirada de embajadores de Madrid (Fernández Liesa, Manero \& Quispe, 2014).

Una posición postergada que no comenzará a suavizarse hasta, al menos, los años cincuenta. En efecto, el 4 de noviembre de 1950, de nuevo por Resolución de la Asamblea General las Naciones Unidas, a propuesta de la República Dominicana y Perú (Fernández Liesa, Manero \& Quispe, 2014), se abriría la puerta a que España fuera incorporándose a las diferentes agencias de la organización -en 1951 ingresa en la FAO y la OMS y en 1952 en la UNESCO- (Fernández Liesa, Manero \& Quispe, 2014). Era la antesala del ingreso de España en las Naciones Unidas en 1955 (Granell, 2011), obtenido con un considerable apoyo: once votos a favor y una abstención en el Consejo de Seguridad y cincuenta y cinco votos a favor, ninguno en contra y dos abstenciones en la Asamblea General (Fernández Liesa, Manero \& Quispe, 2014). ${ }^{2}$

Sería precisamente la presencia en Naciones Unidas lo que permitiría a España ingresar en otras organizaciones internacionales, de carácter económico fundamentalmente como la OECE, el FMI, el Banco Mundial o la UNCTAD (Granell, 2011).

\section{La presencia española en mecanismos multilaterales después de la transición a la democracia}

Si en el periodo anterior la nota dominante había sido la tímida presencia española en los mecanismos multilaterales, aunque en ocasiones no tanto por falta de voluntad de estar presentes como porque las características del sistema político entonces existente no lo permitían, en la fase posterior a la transición la nota característica será no sólo la creciente participación en mecanismos de este tipo de mecanismos sino la consideración de que, de forma ineludible, el multilateralismo debía ser uno de los elementos que caracterizaran a la política exterior. Un planteamiento que, si se quiere, con la única excepción del periodo 2002-2004, se ha mantenido inalterable a pesar de los cambios de gobierno (Arenal, 2008) hasta la actualidad y que explica que España hoy forma parte de todas las grandes organizaciones internacionales sino el décimo estado del mundo en función del número de organizaciones internacionales en las que participa y el decimosexto si el criterio es el número de sedes de organizaciones internacionales con que cuenta en su territorio (Granell, 2011).

Así, ya en 1975, España firmaría el Acta Final de Helsinki y, sólo dos años después, en 1977, sería admitida en el Consejo de Europa (Granell, 2011), algo especialmente importante por lo que, al menos en teoría, significa la participación en esta organización en relación con la garantía de derechos y libertades y porque España aún no contaba con una Constitución escrita. Un ingreso coherente con la ratificación a que España procede entre ese año, 1977, y 1980 de los dos pactos de Derechos Civiles y Políticos y de Derechos Económicos, Sociales y Culturales (Fernández Liesa, Manero, \& Quispe, 2014).

Ya en 1982, la incorporación a la Organización del Tratado del Atlántico Norte (OTAN) supuso un paso más en el retorno de España a Europa, a las estructuras de seguridad occidental y, en definitiva a la sociedad internacional. Una incorporación que, no obstante no fue completa, pues España permanecería, por el momento fuera de la Estructura Militar de la OTAN.
La presencia en Maciones Unidas permitiría a España ingresar en otras Organizaciones Intemacionales 
Con todo, el episodio clave de este periodo se produce probablemente el 1 de enero de 1986, apenas diez años después del inicio de la transición española, en el momento en que se consuma la incorporación de España a las Comunidades Europeas. Era la culminación de una aspiración española de larga data y un camino que se inicia en 1977 cuando tras la muerte de Franco, en el marco de la transición a la democracia liderada por el gobierno de Adolfo Suarez y con el apoyo de una opinión pública claramente europeísta, el 27 de julio, el entonces Ministro de Asuntos Exteriores, Marcelino Oreja presentó las cartas correspondientes solicitando que se celebraran negociaciones para el ingreso de España en la CEE, la CECA y la CEEA (Granell, 2011 ) y que fue posible tanto gracias a los cambios vividos por España, a los cuales acabamos de referirnos, como a la propia voluntad de las Comunidades Europeas de expandir su modelo basado en el gobierno y los valores democráticos (Moreno, 2010).

En lo sucesivo, si algo caracterizaría a España sería una cada vez más activa participación en el proceso de construcción comunitaria en general y en las conferencias intergubernamentales en particular. En este sentido, tendría un papel destacado tanto en el laborioso proceso de la "non nata" Constitución Europea, como en los esfuerzos por lograr que los avances previstos en dicho texto acabaran por plasmarse, al menos en cierta medida, en el Tratado de Lisboa. Por lo demás, esa clara implicación española en el proceso de construcción europea se vería acompañada y, si se quiere, reforzada por la presencia de españoles en posiciones clave en las comunidades a la que haremos referencia con detalle más adelante.

En esa misma línea de proactividad, España hizo importantes esfuerzos por estar entre los estados que participaran desde un primer momento en la puesta en marcha de la moneda única, el Euro y, cuenta con un Embajador en Misión Especial para las relaciones con las Instituciones Europeas, Enrique González.

En otro orden de cosas, es innegable que, en términos estrictamente económicos, España se ha beneficiado enormemente de su pertenencia a las Comunidades Europeas. Baste decir al respecto que ha sido un receptor neto hasta la ampliación al este y que, desde ese momento la situación no ha sido desfavorable para España sino que ha quedado en equilibrio. De este modo, solo a partir de 2014 podría empezar España a ser contribuyente neto a la UE.

Aunque se trata de una organización menor en comparación con las mencionadas hasta este punto, el mapa de la creciente presencia española en organizaciones internacionales no estaría completo si no mencionamos el ingreso de España en la Unión Europea Occidental, a la que se incorpora como observadora en 1988 y, en 1990 como miembro de pleno derecho.

El cambio del partido gobernante que, entre los años 1996-2002 sería el Partido Popular liderado por José María Aznar, no supuso modificación alguna de la clara apuesta por hacer del multilateralismo uno de los elementos característicos de la política exterior española. Consecuencias de ello serán la continuidad que se produce en esos años en lo que hace a la progresiva integración de España en la OTAN, que culminaría con la incorporación a la estructura de mandos en 1996, la actitud claramente positiva en relación con el proceso de integración europea, puesta de manifiesto en la firme voluntad de que España cumpliera los criterios de convergencia y pudiera así participar en la moneda única desde un primer momento (Arenal, 2008) o el cuarto de los bienios en que España ha contado con un representante no permanente en el Consejo de Seguridad de las Naciones Unidas, el de los años 2003-2004³. 
Otro tanto cabe decir de la creciente participación española en cumbres de jefes de Estado y de Gobierno de diverso tipo (Priego, 2014), formato crecientemente prevalente en la diplomacia del siglo XXI (Rojas). Entre ellas, cabe mencionar, por la relevancia del área presente en ellas, las cumbres ASEM (Asia-Europe Meetings en sus siglas en inglés), máximo exponente, en realidad de un proceso multilateral más amplio o las cumbres Ibero-Americanas, que reúnen a los jefes de Estado y de Gobierno de los estados de América Latina, España y Portugal y que España se ha esforzado especialmente en impulsar por tratarse de un área geográfica preferente en su política exterior (Priego, 2014). Prueba de ese esfuerzo es la figura del Embajador en Misión Especial para las Cumbres Iberoamericanas, mantenida por los últimos gobiernos con independencia de su color político y que demuestra su compromiso con estos eventos ${ }^{4}$.

Junto a ello, también cabe mencionar la presencia de España en las llamadas "formaciones G". En concreto, España participaría, por primera vez, en una reunión del G-8 en 2002 por estar ejerciendo la presidencia de turno de la UE (Granell, 2011).

Una línea, la de la apuesta por el multilateralismo, que retomaría, tras el breve paréntesis de los años 2002-2004 al que nos hemos referido más arriba, el gobierno liderado por José Luis Rodríguez Zapatero. En esa línea, se relanzaría la apuesta por la implicación decidida y sin fisuras de España en el proceso de construcción europea y se situaría a las Naciones Unidas como uno de los pilares básicos para la política exterior española.

Mención especial merece en esta línea también, el compromiso del gobierno del Partido Socialista Obrero Español liderado por José Luis Rodríguez Zapatero, que en este caso no difiere sustancialmente del que ya había expresado su antecesor, el gobierno del Partido Popular, con los "Objetivos del Milenio" establecidos por la Asamblea General de Naciones Unidas.

Del mismo modo, y en esa línea de continuidad, España sería invitada, por primera vez, a la reunión del G-20 celebrada en 2008 (Granell, 1036).

Por otra parte, a partir de esos años, el compromiso con el multilateralismo se refleja en el hecho de que, progresivamente, España no se limita a participar en organizaciones y procesos multilaterales existentes sino que trata de potenciarlos o de fomentar la creación de otros nuevos. Así, España será, junto con las propias Naciones Unidas (por medio del entonces Secretario General de la Organización, Kofi Annan) o Turquía (de la mano de su primer Ministro, Recep Tayyip Erdogan), uno de los promotores de la "Alianza de Civilizaciones". Prueba de su compromiso con esta iniciativa fue el nombramiento de un Embajador en Misión Especial para la Alianza de Civilizaciones, puesto que ocupó Belén Alfaro. En una línea similar, en el marco del proceso ASEM antes mencionado, también será España impulsora de una iniciativa específica en relación con el diálogo interreligioso. Junto a todo ello, en una muestra más de esa apuesta por el diálogo religioso, también se nombraron Embajadores en Misión Especial para las relaciones tanto con comunidades judías como con la musulmana 5 .

De la misma manera, España será uno de los estados que se hagan eco de la importancia de lograr un multilateralismo eficaz (Arenal, 2008), un planteamiento que va unido a su defensa del papel de las Naciones Unidas y de la importancia del respeto al Derecho Internacional. Así por

4 En la actualidad es Diego Bermejo Romero de Terreros está acreditado con esta figura.

5 En la actualidad Ignacio Rupérez es Embajador en Misión Especial encargado de las relaciones con las comunidades y organizaciones musulmanas en el exterior. Hasta febrero de 2014 Álvaro Albacete fue Embajador en Misión Especial encargado de las relaciones con las comunidades y organizaciones judías en el exterior

\section{España no se limita a participar en procesos multilaterales existentes, sino que fomenta la creación de otros nuevos}


ejemplo, S. M. el Rey Juan Carlos I expresaría este compromiso español con el multilateralismo eficaz en varias ocasiones, entre ellas, en 2005, en su intervención ante la ONU (Santamaría, 2005). Asimismo, una muestra de estos planteamientos sería el lanzamiento de una Estrategia Global contra el terrorismo impulsada por España en el marco de las Naciones Unidas, una iniciativa en la que cuenta incluso con el apoyo de los miembros del Consejo de Seguridad (Lavrov, Moratinos, 2007). Por su parte, el pasado mes de septiembre, también el Presidente del Gobierno Mariano Rajoy, intervino ante la Asamblea General de Naciones Unidas con el fin de solicitar oficialmente un asiento para España en el Consejo de Seguridad como miembro no permanente para los años 2015-2016 (Santos, 2013).

Y es que, como ya ocurriera con cambios anteriores en lo que respecta al partido gobernante, el ascenso al poder del Partido Popular tras las elecciones celebradas en noviembre de 2011 no supuso, contrariamente a lo que algunos planteaban, un cambio de gran calado en la política exterior española (Manrique, 2012). Tal ausencia de cambios se reflejó no sólo en el ámbito de los objetivos sino también en el de los instrumentos y más en concreto y, por lo que a este trabajo se refiere, en lo que hace al papel del multilateralismo entre los instrumentos españoles de política exterior y, en consecuencia, a la participación española en organizaciones, mecanismos $\mathrm{y}$ foros de distintos tipos.

La primera muestra de continuidad en este sentido fue, de hecho, para muchos, el nombramiento de José Manuel García Margallo como Ministro de Asuntos Exteriores. En efecto, el hecho de apostar por una persona con una larga trayectoria como eurodiputado para ese cargo parecía indicar ya desde el principio la continuidad de la apuesta por la Unión Europea, incluso en un momento de dificultad marcado por la crisis económica global y sus consecuencias, particularmente graves para los estados de la UE y aún más para algunos de ellos, entre los que se encontraba España. En esa línea, el nuevo ministro no tardaría en afirmar que "la Unión Europea será la primera preocupación” (Ministerio de Asuntos Exteriores, 2011).

A modo de resumen, la siguiente tabla recoge la participación de España en las organizaciones internacionales de las que ha sido o es miembro de pleno derecho:

Tabla 1. Organizaciones Internacionales en las que España es o ha sido parte.

\begin{tabular}{|c|c|c|c|c|c|}
\hline Organización & Ingreso & Sede & $\begin{array}{l}\text { Embajador/es } \\
\text { Actual }\end{array}$ & Desde & Observaciones \\
\hline Sociedad de Naciones & 1919 & Ginebra & & & \\
\hline OIT & 1919/1956 & Ginebra & & & $\begin{array}{l}\text { España se integró en } \\
1919 \text { para abandonarla } \\
\text { en } 1939 \text {. Volvió con su } \\
\text { entrada en la ONU. }\end{array}$ \\
\hline \multirow[t]{3}{*}{ Naciones Unidas } & 1955 & Nueva York & Fernando Arias & 2012 & \\
\hline & & Ginebra & Ana $\mathrm{M}^{\mathrm{a}}$ Menéndez & 2012 & \\
\hline & & Viena & Carmen Buján & 2010 & \\
\hline $\begin{array}{l}\text { Organización Internacional } \\
\text { para las Migraciones }\end{array}$ & $\begin{array}{l}1958 / 1979 \\
\text { y } 2006\end{array}$ & Ginebra & & & $\begin{array}{l}\text { En } 1979 \text { España pasó } \\
\text { a ser observador para } \\
\text { volver a ser miembro en } \\
2006\end{array}$ \\
\hline OCDE & 1961 & París & $\begin{array}{l}\text { Ricardo Díez- } \\
\text { Hochleitner }\end{array}$ & 2011 & \\
\hline OSCE & 1973 & Viena & Fernando Valderrama & 2011 & \\
\hline $\begin{array}{l}\text { Organización Mundial del } \\
\text { Turismo }\end{array}$ & 1975 & Madrid & & & $\begin{array}{l}\text { En } 1976 \text { se incorporó al } \\
\text { sistema de NNUU }\end{array}$ \\
\hline Consejo de Europa & 1977 & Estrasburgo & $\begin{array}{l}\text { Fernando } \\
\text { Alvargonzález }\end{array}$ & 2011 & \\
\hline
\end{tabular}




\begin{tabular}{|c|c|c|c|c|c|}
\hline OTAN & 1981 & Bruselas & $\begin{array}{l}\text { Miguel Aguirre de } \\
\text { Cárcer }\end{array}$ & 2013 & \\
\hline $\begin{array}{l}\text { Organización de Estados } \\
\text { Iberoamericanos para la } \\
\text { Educación, la Ciencia y la } \\
\text { Cultura }\end{array}$ & 1985 & Madrid & & & \\
\hline Unión Europea & 1986 & Bruselas & Alfonso Dastis & 2011 & \\
\hline Unión Europea Occidental & 1990 & París & Alfonso Dastis & 2011 & $\begin{array}{l}\text { Primero fue admitida } \\
\text { como Observador en } \\
\text { 1988. Desde } 2011 \\
\text { integrada en la UE }\end{array}$ \\
\hline $\begin{array}{l}\text { Tribunal Internacional de } \\
\text { derecho del mar }\end{array}$ & 1994 & Hamburgo & & & \\
\hline $\begin{array}{l}\text { Organización Mundial del } \\
\text { Comercio }\end{array}$ & 1995 & Ginebra & & & \\
\hline
\end{tabular}

FUENTE: Elaboración propia usando varias fuentes

La cada vez más decidida apuesta de España por el multilateralismo, ha ido acompañada, como no podría ser de otro modo, de una creciente presencia de españoles en posiciones clave en las distintas organizaciones internacionales. Es el caso del Embajador Jaime de Piniés que sería presidente de la Asamblea General de Naciones Unidas en los años 1985-86; Marcelino Oreja, Secretario General del Consejo de Europa entre 1984 y 1989; José Mª de Areilza, Presidente de la Asamblea Parlamentaria de 1981 a 1983, Miguel Ángel Martínez o Luis Maa de Puig que también ocuparon ese puesto de 1992 a 1996 y de 2008 a 2011 respectivamente, Federico Mayor Zaragoza, Secretario General de la UNESCO de 1987 a 1999 (El País, 2011) o Javier Rupérez Presidente de la Asamblea Parlamentaria de la OSCE de 1996 a 1998 y Secretario General Adjunto y Director Ejecutivo del Comité Contraterrorista del Conejo de Seguridad entre 2004 y 2007 (Priego, 2014). Asimismo cabe mencionar a Álvaro Gil Robles, primer Comisario Europeo de Derechos Humanos del Consejo de Europa, en los años 1999-2006 y Presidente del Comité de Ministros del Consejo de Europa durante el bienio 2008-2009 (Granell, 2011), a José Borrell, Presidente del Parlamento Europeo entre 2004 y 2007, a Enrique Barón, que presidió la misma institución durante los años 1989-1992) o a Joaquín Almunia, comisario y vicepresidente de la Comisión Europea entre 2009 y 2014 (El País, 2011). La lista estaría incompleta si no mencionáramos a Javier Solana, Secretario General de la OTAN entre 1995 y 1999 y Mr. PESC entre 1999 y 2009, a Rodrigo Rato, Director Ejecutivo del FMI entre 2004 y 2007, a Ana Palacio, vicepresidenta del Banco Mundial entre 2006 y 2008, al General José Julio Rodríguez, que presidió el Comité Militar de la OTAN en 2008 (Granell, 2011) así como a Joan Clos, director ejecutivo de ONU-Habitat (El País, 2011).

Tabla 2. Españoles en puestos destacados en Organizaciones Internacionales

\begin{tabular}{|l|l|l|l|}
\hline Nombre & Organización & Puesto & Fecha \\
\hline Salvador de Madariaga & Sociedad de Naciones & $\begin{array}{l}\text { Jefe del Departamento de Desarme de la Sociedad } \\
\text { de Naciones }\end{array}$ & $1922-1927$ \\
\hline José María Areilza & Consejo de Europa & Presidente de la Asamblea Parlamentaria & $1987-1983$ \\
\hline Marcelino Oreja & Consejo de Europa & Secretario General & $1984-1989$ \\
\hline Jaime de Piniés & ONU & Presidente de la Asamblea General & $1985-1986$ \\
\hline Federico Mayor Zaragoza & UNESCO & Secretario General & $1987-1999$ \\
\hline Enrique Barón & UE & Presidente del Parlamento Europeo & $1989-1992$ \\
\hline
\end{tabular}




\begin{tabular}{|c|c|c|c|}
\hline Miguel A. Martínez & Consejo de Europa & Presidente de la Asamblea Parlamentaria & $1992-1996$ \\
\hline Javier Solana & OTAN & Secretario General & 1995-1999 \\
\hline Javier Rupérez & OSCE & Presidente de la Asamblea Parlamentaria & 1996-1998 \\
\hline Miguel A. Moratinos & UE & Alto Representante para Oriente Medio & $1996-2003$ \\
\hline Carlos Westendorf & ONU & $\begin{array}{l}\text { Alto Representante Internacional para Bosnia y } \\
\text { Herzegovina }\end{array}$ & $1997-1999$ \\
\hline José María Gil Robles & UE & Presidente del Parlamento Europeo & 1997-1999 \\
\hline Álvaro Gil Robles & Consejo de Europa & $\begin{array}{l}\text { Primer Comisario Europeo para los Derechos } \\
\text { Humanos }\end{array}$ & 1999-2006 \\
\hline Javier Solana & UE & Alto Representante del Consejo para la PESC & $1999-2009$ \\
\hline Rafael Estrella & OTAN & Presidente de la Asamblea Parlamentaria & $2000-2002$ \\
\hline Ana Palacio & Banco Mundial & Vicepresidenta & $2002-2008$ \\
\hline Rodrigo Rato & $\begin{array}{l}\text { Fondo Monetario } \\
\text { Internacional }\end{array}$ & Director Gerente & $2004-2007$ \\
\hline Josep Borrell & UE & Presidente del Parlamento Europeo & $2004-2007$ \\
\hline Javier Rupérez & ONU & $\begin{array}{l}\text { Director Ejecutivo del Comité contra el Terrorismo } \\
\text { del Consejo de Seguridad de las Naciones Unidas }\end{array}$ & $2004-2008$ \\
\hline Luis María de Puig & Consejo de Europa & Presidente de la Asamblea General & 2008-2011 \\
\hline Joan Clos & ONU & $\begin{array}{l}\text { Director Ejecutivo del Programa de Naciones Unidas } \\
\text { para los Asentamientos Humanos }\end{array}$ & 2010-Hoy \\
\hline Bernardino León & UE & Representante Especial para el Mediterráneo Sur & 2011-hoy \\
\hline Álvaro Albacete & ONU & $\begin{array}{l}\text { Asesor del Secretario General para el Diálogo } \\
\text { Religioso en KAICIID (International Centre } \\
\text { Interreligious and Intercultural Dialogue) }\end{array}$ & 2014-Hoy \\
\hline
\end{tabular}

FUENTE: Elaboración propia usando varias fuentes.

Por el contrario, y en fuerte contraste con esa presencia relativamente importante de españoles en posiciones de relevancia, encontramos, sin embargo, el reducido número de funcionarios internacionales españoles presentes en las diferentes organizaciones internacionales. Así, según datos de la Asociación Española de Funcionariado Internacional serían, en la actualidad, solamente unos 1000 españoles los que tienen este tipo de ocupación. Como señala Manuel Montobbio, en buena medida, esa baja presencia es consecuencia de la tardía incorporación de España a muchas organizaciones de la que hemos estado hablando. Sin embargo, como este autor plantea sólo en la medida en que la opinión pública y la ciudadanía en general, pero también las universidades, las empresas, los medios de comunicación y las ONGs tengan interés en las organizaciones internacionales, éstas serán contempladas como una verdadera opción profesional por quienes estén al inicio de su carrera y se podrá revertir la situación. (Montobbio, 2006).

\section{Los objetivos de la política exterior española y la participación en mecanismos multilaterales}

Toda política supone la articulación de una serie de medios para conseguir determinados fines y la política exterior española no es, como apuntábamos más arriba, una excepción. Por ello si no se cuenta con medios suficientes para lograr una presencia e influencia internacionales de cierta consideración, se corre el riesgo de generar una situación de pérdida de confianza a nivel interno y de pérdida de prestigio a nivel externo (Pérez Gil, 2012, 131). Esta realidad es bien visible en el caso de España, para la que los periodos más exitosos han sido también épocas 
de mayor presencia internacional en tanto que en las etapas más sombrías ha visto reducirse aquella considerablemente.

En consecuencia y, para evitar semejante escenario, si no se cuenta con demasiados medios en términos de poder duro -y tal parece ser el caso de España-, lo más razonable parece apostar por el poder inteligente y combinar hábilmente instrumentos de poder blando y duro o, en otras palabras, y siguiendo al Profesor Pérez Gil, medios pacíficos y coactivos de política exterior (Pérez Gil, 2012, 131) como instrumentos para lograr influencia internacional y conseguir los objetivos planteados.

En lo que hace a los objetivos españoles en materia de política exterior hay que decir que su identificación ha sido desde siempre una tarea que no cabe calificar sino de ardua, aunque no es menos cierto que en los últimos tiempos han ido apareciendo algunos documentos que, pese a su ambigüedad, su carácter político y -unido a este último- su frecuente tono declarativo, arrojan cierta luz sobre la cuestión.

Entre tales documentos cabe destacar el informe "Hacia una renovación estratégica de la política exterior española” elaborado este mismo año por el Real Instituto Elcano. En él se establecen con cierta claridad los objetivos españoles en política exterior -democracia, seguridad, competitividad y talento, integración europea, responsabilidad internacional e influencia- (Molina, 2014; Diario de Navarra, 2014).

Si bien es cuestionable si la distinción entre objetivos e instrumentos es todo lo clara que sería deseable, el documento también arroja luz en lo que hace a los medios de la política exterior, identificando como instrumentos susceptibles de ser utilizados para alcanzar los objetivos - diplomacia, protección y asistencia consular, seguridad, defensa e inteligencia, economía y promoción empresarial, cooperación al desarrollo y acción cultural y educativa científica- (Molina, 2014; Diario de Navarra, 2014).

En este sentido, en tanto en cuanto la diplomacia se considera uno de los instrumentos para alcanzar los objetivos españoles en materia de política exterior, el papel de los mecanismos multilaterales como instrumento en la política exterior española queda claro, pues tales mecanismos son, entre otras cosas, mecanismos de diplomacia, diplomacia multilateral, pero diplomacia al fin y al cabo.

Por otra parte, el propio informe plantea como uno de los niveles de actuación en que trabajar para alcanzar los objetivos planteados, precisamente, el nivel multilateral (Molina, 2014)

Dicho de otro modo, España se decanta por un mayor peso de los medios pacíficos, en particular la diplomacia, la negociación y la participación en organizaciones (Pérez Gil, 2012, 131) como elementos que le puedan ayudar a generar su poder inteligente, algo razonable pues, como señala este autor, la participación en mecanismos multilaterales favorece precisamente a aquellos estados que son potencias medianas, pues les ofrecen la oportunidad de unir sus fuerzas frente a las preferencias de las grandes potencias (Pérez Gil, 2012, 135)

Un poder inteligente que pueda situarla en una posición favorable para la consecución de algunos de sus objetivos, esto es, en términos de favorecer la democracia, la seguridad, la competitividad y el talento, pero que también le permita contribuir al avance y profundización de la integración europea, mostrar su responsabilidad y compromiso en el mantenimiento de la sociedad internacional y contar con una creciente influencia en los asuntos internacionales. 


\section{Conclusión}

En este sentido, parece claro que la apuesta por el multilateralismo ha contribuido a fomentar el protagonismo internacional de España a la que, gracias a él se ve como un actor preocupado por lo que ocurre en el contexto internacional, con unas claras señas de identidad y que mantiene una actitud proactiva (Arenal, 2008) y no reactiva respecto de los asuntos internacionales.

Esta contribución es tanto más importante si tenemos en cuenta que, tradicionalmente, los periodos más exitosos de la historia de España han sido también épocas en que ha tenido una mayor presencia internacional en tanto que ésta frecuentemente se ha visto reducida en los más sombríos.

En consecuencia, España debe mantener e incrementar-como ha venido haciendo-su presencia en los distintos foros multilaterales tratando de influir en su agenda y en sus decisiones (García Encina, 2011) y buscando que éstas sean, en la medida de lo posible, acordes a sus objetivos en materia de política exterior y coherentes con los otros instrumentos empleados en la misma.

\section{Bibliografía}

Arenal, C. (2008). Consenso y Disenso en la Política Exterior de España. Documento de TrabajoReal Instituto Elcano, 25, 2 de junio.

Santamaría, O. (2014, 15 de septiembre). El Rey destaca el compromiso 'firme’ de España con el multilateralismo. Diario de León. Disponible en http://www.diariodeleon.es/noticias/ afondo/rey-destaca-compromiso-firme-espana-multilateralismo_217556.html Con acceso 12 de mayo de 2014

(2014, 23 de febrero). España debe presentarse como una potencia media en su política exterior. Diario de Navarra. Disponible en www.diariodenavarra.es Con acceso 24 de junio de 2014

(201 1, 24 de junio). España pugna por ascender de la segunda división en los organismos internacionales. El País.Disponible en http://politica.elpais.com/politica/2011/06/24/actualidad/1308942121_722277.html Con acceso 12 de mayo de 2014

Fernández Liesa, C, Manero Salvador, A., \& Quispe, F. (2014). España y las OI. Disponible en http://ocw.uc3m.es/periodismo/periodismo-internacional-ii/material-de-clase-1/leccion_10.pdf Con acceso 26 de julio de 2014

García Encina, C. (2011). España 2012: el cambio de las organizaciones internacionales. Grupo de Estudios Estratégicos, 8942, 13 de octubre.

Granell, F. (2011). España y las Organizaciones Internacionales, en Beneyto, J. M. y Pereira, J. C. (Dirs.). Política Exterior Española: Un balance de futuro. Madrid: Biblioteca Nueva

Lavrov, S. y Moratinos, M. A. (2007, 9 de febrero). España, Rusia y el Multilateralismo. El País. Disponible en http://elpais.com/diario/2007/02/09/opinion/1170975605_850215.html Con acceso 12 de mayo de 2014

Manrique, M. (2012). Claves de la política exterior española: enero-marzo 2012. Policy Brief FRIDE, 76. 
Molina, I. (coord.) (2014). Hacia una renovación estratégica de la Política Exterior Española. Madrid: Real Instituto Elcano.

Montobbio, M. (2006). Instituciones para una cooperación internacional al desarrollo de calidad. Revista CIDOB d'Affers Internationals, 72, Diciembre 2005-Enero 2006, 85-95.

Moreno Juste, A. (2010). Por fin Europa: la transición y el camino hacia la adhesión a la CEE, en Forner, S. (Ed.). Coyuntura Internacional y Política Española (1898-2004). Madrid: Biblioteca Nueva.

Nye, J. Jr. (2011). The Future of Power. Nueva York: Public Affairs

Pérez Gil, L. V. (2012). Elementos para una teoría de la política exterior. Valencia: Tirant lo Blanch

Priego, A. (2014). Spanish soft power and its structural (non-traditional) model of diplomacy en García, D. \& Pacheco, R. Contemporary Spanish Foreign Policy. Abingdon: Routledge.

Rojas, F. (2010). El difícil camino hacia un multilateralismo cooperativo, proactivo y eficaz. Diplomacia de Cumbres, Secretaría General FLACSO, 1-8.

Santos, P. (2013, 26 de septiembre). Rajoy ondea la bandera del multilateralismo. El Periódico. Disponible en http://www.elperiodico.com/es/noticias/internacional/rajoy-ondea-bandera-del-multilateralismo-2212487 Acceso 12 de mayo de 2014 